\title{
Efeitos pleiotrópicos das estatinas na sepse
}

\section{Pleiotropic effects of statins in sepsis}

Silvana Menegoto Nogueira Di Giusto¹, Kelly Cristina Batista de Souto Queiroz¹, Rodrigo Daminello Raimundo², Luiz Carlos de Abreu Monica Akemi Sato ${ }^{1}$

${ }^{1}$ Curso de Fisiologia Humana da Faculdade de Medicina do ABC (FMABC) - Santo André (SP), Brasil.

${ }^{2}$ Centro Universitário Sant'Anna, São Paulo (SP), Brasil; Universidade Cidade de São Paulo (UNICID) - São Paulo (SP), Brasil. Faculdades Metropolitanas Unidas (FMU) São Paulo (SP), Brasil.

DOI: http://dx.doi.org/10.7322/abcshs.v39i1.249

\section{RESUMO}

O objetivo do presente estudo foi revisar os efeitos da terapia com estatinas em pacientes com quadro de sepse. Foi realizada uma busca bibliográfica de artigos na base de dados MEDLINE/PubMed, SciELO e LILACS, no período de publicação delimitado entre 2009 e 2013. Foram incluídos artigos referentes a ensaios clínicos randomizados controlados e estudos de coorte observacionais clínicos. Foram encontrados diversos estudos clínicos e observacionais que investigaram o efeito do uso de estatinas em infecções e em sepse, tanto de uso contínuo pré-hospitalar (com ou sem interrupção durante internação) ou com início imediato pós-internação. Alguns estudos descrevem prováveis efeitos positivos e benéficos da terapia com estatinas no quadro de sepse, dentre eles a melhora dos parâmetros inflamatórios e da taxa de mortalidade, porém, esses resultados não se sustentam quando são aplicados métodos estatísticos que levam em conta diferentes variáveis, tais como idade, sexo, comorbidades e gravidade da doença. Até o momento nenhum estudo demonstrou evidências sólidas e significativas quanto à redução de mortalidade e morbidade no quadro séptico associado ao uso de estatinas, indicando que seu efeito benéfico e protetor ainda não foi plenamente delimitado, sendo necessários mais estudos que confirmem os resultados até agora encontrados.

Palavras-chave: sepse; anticolesterolemiantes; choque séptico; hidroximetilglutaril CoA redutases; inflamação.

\section{ABSTRACT}

The objective of this study was to review the effects of statin therapy in patients with signs of sepsis. A bibliographic search of articles published between 2009-2013 was performed in the MEDLINE/PubMed, SciELO and LILACS databases. Randomized controlled trials and observational clinical cohort studies were included. The results show that several clinical and observational studies have investigated the effect of statin in infection and sepsis, both pre-hospital continuous use (with or without interruption during hospitalization) or starting immediately post-hospitalization. Some studies describe positive and beneficial effects of statin therapy in the context of sepsis, including improvement on inflammatory parameters and mortality rates. However, these results do not hold on when statistic methods, which take into account different variables such as age, sex, comorbidities and severity disease, are applied. To date, no study has demonstrated strong and significant evidence regarding the reduction of morbidity and mortality in sepsis associated with the use of statin. This indicates that beneficial and protective effects have not been fully defined yet. More researches are required to confirm the results found so far.

Keywords: sepsis; anticholesteremic agents; shock, septic; hydroxymethylglutaryl CoA reductases; inflammation.

Recebido em: 11/09/2013

Revisado em: 28/02/2014

Revisado em: 13/03/2014 


\section{INTRODUÇÃO}

Sepse é uma síndrome caracterizada por um conjunto de manifestações graves em todo o organismo, desencadeadas em resposta a alguma agressão advinda de uma infecção ${ }^{1,2}$. Em geral, o diagnóstico infeccioso se resume a um órgão ou sistema, mas é suficiente para causar um processo inflamatório em todo o organismo $^{2}$. Pode apresentar múltiplas manifestações, dentre elas a disfunção ou a falência de um ou mais órgãos. Se houver uma associação da sepse com a disfunção orgânica, hipotensão ou hipoperfusão, denomina-se sepse grave; se a sepse for associada à hipotensão arterial persistente refratária à reposição volêmica, redução da perfusão, acidose lática, oligúria e alteração do estado mental, então se denomina choque séptico ${ }^{2,3}$. Sendo um dos mais significativos problemas de saúde pública, a sepse é uma das principais causas do aumento da morbidade e da mortalidade em pacientes em UTI ${ }^{4-6}$. Apresenta alarmantes incidência e taxa de mortalidade, além dos custos estimados para o tratamento desses pacientes serem cada vez mais elevados ${ }^{7}$. Em todo o mundo, cerca de 18 milhões de casos de sepse grave ocorrem anualmente e, dentre esses pacientes, a taxa média de mortalidade intra-hospitalar é de $36,2 \%{ }^{8}$.

Diversos mecanismos permeiam sua fisiopatologia, tais como produção de citocinas inflamatórias, adesão e migração de monócitos e células T, produção de óxido nítrico e apoptose. Na sepse, a cascata da produção de citocinas (reguladores e mediadores de respostas imunológicas) é desencadeada por diversos fatores como a endotoxina, o recrutamento leucocitário e a produção de substâncias biologicamente ativas ${ }^{3,4,8-10}$. As endotoxinas constituem a maior porção da membrana externa de bactérias gram-negativas ${ }^{9}$, gerando potente ativação macrofágica mesmo em baixas concentrações, e são considerados os mediadores primários da síndrome séptica e suas complicações ${ }^{11}$. Na circulação sistêmica, essas moléculas podem causar desequilíbrio hemodinâmico, disfunção orgânica e podem levar à apoptose em células linfoides e mieloides, necrose e morte celular ${ }^{12}$.

Alguns fatores imunogenéticos e moleculares têm sido relacionados à fisiopatologia da sepse, tais como a hereditariedade e a expressão gênica nas diversas fases da doença. As primeiras evidências da participação da hereditariedade nas síndromes infecciosas mostraram uma maior incidência de sepse nos filhos de pais que morreram de infecções. Por mais de uma década tem havido considerável interesse na possibilidade de variações genéticas influenciarem a vulnerabilidade de indivíduos às infecções ${ }^{13}$.

Condutas clínicas adotadas para o tratamento da sepse são, predominantemente, voltadas para o suporte da vida e controle do agente agressor. Apesar das diversas estratégias adotadas para seu combate, como o uso de antimicrobianos, compensações cardiovasculares e hemodinâmicas e terapia imunomoduladora ${ }^{13}$, nem sempre há o resultado eficaz esperado. Na sepse ocorre uma desregulação no processo inflamatório, que é caracterizado pela liberação não balanceada de mediadores pró e anti-inflamatórios. Essa desregulação da resposta inflamatória em pacientes sépticos, associada a fatores como organismos enfraquecidos, debilitados ou com defesas imunológicas insuficientes, tem sido implicada no desenvolvimento da síndrome da disfunção de múltiplos órgãos, choque séptico e óbito ${ }^{14,15}$, sendo assim, a sepse ainda continua sendo considerada um estado de saúde grave e de grande risco ao paciente.

As estatinas são fármacos utilizados frequentemente nos tratamentos de hipercolesterolemia e contribuem também na prevenção primária e secundária de eventos cardiovasculares, modulando o processo arterosclerótico. $\mathrm{O}$ impacto dessas drogas, no entanto, não pode ser explicado apenas pela melhora do perfil lipídico ${ }^{16,17}$, pois ao inibirem a enzima 3-hidroxi-3-metilglutaril coenzima A (HMG-CoA) redutase, limitadora da velocidade na biossíntese do colesterol, as estatinas bloqueiam a síntese de outros produtos dessa reação. Esse bloqueio catalisa a conversão da HMG-CoA ao ácido melavônico, um precursor do colesterol $^{18}$. As estatinas bloqueiam a síntese de outros produtos dessa reação, como os isoprotenoides intermediários geranilgeranilpirofosfato (GGPP) e farnesilpirofosfato (FPP) ${ }^{19}$. O GGPP e o FPP atuam como ativadores de certas proteínas sinalizadoras intracelulares, responsáveis pelo desencadeamento da cascata da inflamação. Entre as proteínas sinalizadoras, a Rho tem papel de destaque, por estar envolvida na via de transcrição do NFkB, fundamental na expressão gênica de inúmeros mediadores inflamatórios ${ }^{20}$ que acabam por resultar em outros efeitos secundários à redução do colesterol.

No cenário clínico, as estatinas atenuam a aterogênese coronariana e as lesões vasculares relacionadas à hipertensão arterial sistêmica ${ }^{21,22}$. Essas drogas possuem uma ação anti-inflamatória, antiproliferativa e imunomodulatória in vitro e in vivo ${ }^{23-25}$. Entre essas ações, elas bloqueiam o processo de transcrição de mediadores inflamatórios, inibindo a expressão de citocinas e quimiocinas $^{26}$, o que leva a uma significativa redução da atividade inflamatória na parede vascular, bem como a uma redução do processo inflamatório sistêmico. Algumas das ações das estatinas incluem: diminuição da expressão de fator tecidual, redução de citocinas inflamatórias pelas células endoteliais, inibição de proliferação de células musculares lisas na placa ateromatosa, ação redutora da agregação plaquetária e inibição da secreção de enzimas inflamatórias por macrófagos e monócitos. Esses outros efeitos das estatinas são conhecidos por ações pleiotrópicas e têm sido largamente estudados e explorados.

Estas ações pleiotrópicas contribuem para os efeitos benéficos sobre a doença cardiovascular, mas também parecem desempenhar um papel em outras doenças autoimunes e inflamatórias. Nesse contexto, considerando a sepse como entidade clínica grave com intenso processo de atividade inflamatória associada e, reconhecendo a capacidade de intervenção das estatinas nessas vias, têm sido realizados estudos clínicos a fim de observar e avaliar os efeitos da terapêutica com estatinas sobre a sepse. Esses estudos procuram observar a redução dos riscos relacionados à 
sepse, principalmente a mortalidade e a progressão da doença, e se há ou não melhora significativa na sobrevida do paciente. Sendo assim, o objetivo deste trabalho foi revisar os estudos clínicos que tratam do efeito da terapia com estatinas em pacientes com quadro de sepse.

\section{MÉTODOS}

A busca dos artigos utilizados neste estudo foi realizada nas bases de dados MEDLINE/PubMed, SciELO e LILACS, por artigos publicados no período compreendido entre 2009 e 2013. Para a busca foram utilizadas as palavras-chave: "sepse", "estatina", "choque séptico", "inibidor HMG-Co A redutase” e "inflamação" (com as respectivas palavras em inglês "sepsis", "statin", "septic shock", "HMG-Co A reductase inhibitor" e "inflammation").

Foram incluídos artigos referentes a ensaios clínicos randomizados e estudos de coorte observacionais clínicos com disponibilidade de texto livre em algum site da Internet, nos idiomas inglês, espanhol ou português.

Para a seleção dos artigos, inicialmente foi realizada a triagem dos títulos relacionados ao tema em questão. A princípio foram selecionados 131 artigos, e dentre aqueles disponíveis foram eliminados os que tratavam de outras patologias que não sepse, os que citavam combinação de outros fármacos associados com estatina, os artigos de revisão, os experimentais, os que tratavam da farmacocinética das estatinas, além de cartas ao editor, resumos e questionários, restando assim, 8 artigos que cobriam os critérios acima mencionados.

Posteriormente, os textos completos foram lidos integralmente e as referências dos estudos selecionados foram revisadas a fim de complementar a pesquisa.

\section{RESULTADOS E DISCUSSÃO}

Os dados foram analisados de forma qualitativa e apresentados na forma de tabela (Tabela 1) com a descrição das seguintes características: autores e ano do estudo, descrição da população, tipo de estudo realizado, protocolo utilizado, variáveis estudadas e resultados.

Diversos estudos clínicos e observacionais investigaram o efeito do uso de estatinas em infecções e em sepse, tanto de uso contínuo pré-hospitalar (com ou sem interrupção durante internação), como com início imediato pós-internação. Alguns estudos descrevem prováveis efeitos positivos da terapia com estatinas no quadro inflamatório da sepse $\mathrm{e}^{27-31}$.

O efeito das estatinas na progressão da sepse com administração imediata e contínua somente após a internação foi investigado em dois estudos ${ }^{27,30}$, nos quais foram selecionados pacientes sépticos para receber o medicamento durante o período de internação e seus efeitos foram comparados ao do grupo placebo. Patel et al. ${ }^{30}$ selecionaram pacientes para receber $40 \mathrm{mg} /$ dia de estatina durante a permanência hospitalar ou em um período máximo de 28 dias. Foi observado que neste grupo houve uma incidência significativamente menor de pacientes com sepse convertendo-se em sepse grave ( $n=2$ pacientes), em comparação com o grupo placebo $(n=12)$, portanto, aparentemente o medicamento interferiu na taxa de progressão da sepse, porém, a taxa de mortalidade não apresentou diferença significativa. Já Novak et al. ${ }^{27}$ selecionaram pacientes para receber $40 \mathrm{mg}$ do fármaco em única dose imediatamente após internação e manutenção diária com 20 mg durante hospitalização. Foi observado que, em comparação ao grupo placebo, não houve diferença significativa na incidência de sepse grave. No entanto, a descoberta mais significativa foi a constatação da redução dos níveis de citocinas pró-inflamatórias nos pacientes

Tabela 1: Estudos que analisaram o efeito da estatina na sepse

\begin{tabular}{|c|c|c|c|c|c|}
\hline Autor/ano & $\mathbf{n}$ & $\begin{array}{l}\text { Tipo de } \\
\text { estudo }\end{array}$ & Protocolo & Desfecho avaliado & Resultado \\
\hline Novak et al., $2009^{27}$ & 83 & PR & $\begin{array}{l}\text { Grupos: estatina somente após } \\
\text { internação e placebo }\end{array}$ & Incidência de sepse ou morte & $\begin{array}{l}\text { Estatina pode estar associada à } \\
\text { redução de citocinas inflamatórias }\end{array}$ \\
\hline Kruger et al., $2011^{28}$ & 150 & PR & $\begin{array}{l}\text { Grupos: estatina pré e pós } \\
\text { internação e placebo }\end{array}$ & $\begin{array}{l}\text { Melhora quadro inflamatório } \\
\text { da sepse }\end{array}$ & $\begin{array}{l}\text { Estatina pré e pós internação não } \\
\text { altera parâmetros inflamatórios }\end{array}$ \\
\hline Nseir et al., $2012^{29}$ & 319 & CER & $\begin{array}{l}\text { Grupos: estatina a longo prazo; } \\
\text { a curto prazo e sem estatina }\end{array}$ & $\begin{array}{l}\text { Mortalidade com estatina a } \\
\text { longo e curto prazo }\end{array}$ & $\begin{array}{l}\text { Incidência de morte menor em } \\
\text { grupo com estatina a longo prazo }\end{array}$ \\
\hline Patel et al., $2012^{30}$ & 100 & PR & $\begin{array}{l}\text { Grupos: estatina somente após } \\
\text { internação e placebo }\end{array}$ & $\begin{array}{l}\text { Melhora quadro inflamatório } \\
\text { da sepse }\end{array}$ & $\begin{array}{l}\text { Estatina pós internação pode } \\
\text { impedir progressão da sepse }\end{array}$ \\
\hline Donnino et al., $2009^{31}$ & 2.036 & $\mathrm{CR}$ & $\begin{array}{l}\text { Grupos: estatina pré e pós } \\
\text { internação; com interrupção pós } \\
\text { internação ou sem estatina }\end{array}$ & Mortalidade hospitalar & $\begin{array}{l}\text { Incidência de morte menor com } \\
\text { uso continuado de estatina }\end{array}$ \\
\hline $\begin{array}{l}\text { Mekontso Dessap } \\
\text { et al., } 2011^{32}\end{array}$ & 44 & CR & $\begin{array}{l}\text { Grupos: estatina pré e pós } \\
\text { internação e com interrupção } \\
\text { pós internação }\end{array}$ & $\begin{array}{l}\text { Melhora quadro inflamatório } \\
\text { da sepse }\end{array}$ & $\begin{array}{l}\text { Estatina pré e pós internação } \\
\text { alterou positivamente parâmetros } \\
\text { inflamatórios }\end{array}$ \\
\hline Williams et al., $2011^{33}$ & 395 & $\mathrm{CP}$ & $\begin{array}{l}\text { Grupos: estatina pré e pós } \\
\text { internação e com interrupção } \\
\text { pós internação }\end{array}$ & Mortalidade hospitalar & $\begin{array}{l}\text { Sem diferença na mortalidade } \\
\text { entre os grupos }\end{array}$ \\
\hline Leung et al., $2012^{34}$ & 592 & $\mathrm{CR}$ & $\begin{array}{l}\text { Grupos: estatina pré e pós } \\
\text { internação e com interrupção } \\
\text { pós internação }\end{array}$ & Mortalidade hospitalar & $\begin{array}{l}\text { Sem diferença na mortalidade } \\
\text { entre os grupos }\end{array}$ \\
\hline
\end{tabular}

n: tamanho da amostra; PR: estudo prospectivo randomizado; CER: estudo clínico epidemiológico retrospectivo; CR: estudo coorte retrospectivo; CP: estudo coorte prospectivo 
tratados com estatina, o que, a princípio, poderia ser traduzido como uma melhora do quadro inflamatório geral. Apesar disso, esse estudo foi considerado pelos autores como sendo de fraca potência por incluir um número pequeno de participantes $(n=83)$. Desta forma, a relevância da redução dos níveis de citocinas exercendo um efeito protetor na sepse e apresentando um possível benefício clínico ainda permanece inconclusivo.

Esses ensaios clínicos controlados se basearam em evidências advindas de estudos retrospectivos e prospectivos observacionais anteriores, demonstrando que pacientes em tratamento com estatina pré e pós-hospitalização tiveram melhores resultados nas taxas de progressão da sepse e sobrevida quando comparados a outros grupos ${ }^{29,31}$

Donnino et al. ${ }^{31}$, em estudo retrospectivo, investigaram a associação entre a terapia com estatinas e a taxa de mortalidade em pacientes internados com suspeita de infecção. Todos os pacientes avaliados já faziam uso do medicamento antes da hospitalização e foram observados os resultados daqueles que tiveram interrupção do tratamento após internação e daqueles que continuaram. $\mathrm{O}$ estudo demonstrou que os pacientes internados que receberam estatina $(\mathrm{n}=474)$ tiveram uma taxa de mortalidade mais baixa $(1,9 \%$; IC95\% 0,6-3,3) do que os pacientes que não receberam durante a internação (4,5\%; IC95\% 3,4-5,4). Esses resultados permaneceram positivos mesmo após análises ajustadas para idade, sexo, comorbidades e severidade da doença. Apesar de ter sido uma análise grande, com acompanhamento de 2.036 pacientes, $\mathrm{o}$ estudo demonstra apenas uma associação, e não uma causalidade, entre a terapia com estatinas e a redução da mortalidade em pacientes internados, e também ressalta que alguns pacientes incluídos no estudo provavelmente apresentavam apenas quadro infeccioso e não necessariamente um quadro séptico.

Os resultados positivos encontrados nos estudos com relação ao uso contínuo ou não da estatina pré e pós-internação, incentivaram Nseir et al. ${ }^{29}$ a irem mais além e investigarem se haveria diferenças significativas relacionadas ao tempo prévio de uso do medicamento. Assim, realizaram estudo clínico e epidemiológico com objetivo de avaliar a taxa de mortalidade entre pacientes sépticos em tratamento com estatina em longo e em curto prazo antes da hospitalização. Os pacientes foram separados em grupos, um com uso de estatina pré-hospitalar em longo prazo (mais de 12 semanas, $n=123$ ) e outro com uso em curto prazo (menos de 12 semanas, $n=35$ ), sendo que os resultados ainda foram comparados com pacientes sem uso de estatina $(n=161)$. O resultado encontrado foi que a sobrevida de pacientes sépticos com uso do medicamento em longo prazo e com uso ininterrupto durante internação foi percentualmente maior do que no grupo que utilizava o fármaco em curto prazo ou que nunca o utilizou, independente da gravidade do quadro infeccioso.

Enquanto alguns estudos como esses apresentaram sinais animadores quanto aos efeitos das estatinas na sepse e na redução da mortalidade, em contrapartida outros não demonstraram tanto otimismo assim.
Mekontso Dessap et al. ${ }^{32}$, Williams et al. ${ }^{33}$ e Leung et al. ${ }^{34}$ realizaram estudos observacionais semelhantes, cujo objetivo foi avaliar a associação entre a terapia com estatinas e a taxa de mortalidade ou evolução clínica dos pacientes com sepse, usuários prévios ou não dessa medicação. Foram incluídos nos estudos pacientes admitidos na internação hospitalar com diagnóstico ou suspeita de infecção. Williams et al. ${ }^{33}$ e Leung et al. ${ }^{34}$ avaliaram pacientes que tiveram morte hospitalar em um período de tempo pré-determinado (30 ou 90 dias, respectivamente); enquanto Mekontso Dessap et al. ${ }^{32}$ acompanharam a evolução clínica dos participantes de seu estudo. Em cada estudo a divisão dos integrantes foi semelhante: os pacientes foram divididos em grupos com estatina pré e pós-hospitalar e aqueles que tiveram a medicação interrompida durante internação, e ainda os que não faziam uso do medicamento. Nos resultados do estudo de Williams et al. ${ }^{33}$, observou-se que no grupo de pacientes que continuaram a estatina no hospital não houve diferença significativa na taxa de mortalidade em comparação com os pacientes que nunca usaram. No entanto, pacientes com interrupção da estatina na internação apresentaram maior risco de morte (OR=3,62; IC95\% 1,47-8,94), em comparação aos pacientes que nunca usaram. Enquanto isso, Leung et al. ${ }^{34}$ relatam em suas observações uma boa melhora no quadro clínico dos pacientes que fizeram uso contínuo do medicamento e uma diminuição de $22 \%$ (análise sem ajustes) no risco de morte nos usuários de estatinas em comparação com os não estatina.

Mekontso Dessap et al. ${ }^{32}$ observaram que o as condições clínicas dos pacientes com uso contínuo durante a internação $(n=44)$ foi significativamente melhor quanto à disfunção dos órgãos e à hemodinâmica. Houve desequilíbrios importantes entre os grupos, com mais infecções hospitalares adquiridas, mais necessidade de cirurgias e uma tendência para choque séptico mais acentuado no grupo onde o uso de estatina foi interrompido. Porém, em todos os três estudos, ao serem feitos os ajustes estatísticos de idade, sexo, comorbidades e gravidade da doença, não houve diferença significativa nas taxas de mortalidade e na evolução clínica progressiva no quadro séptico entre os diferentes grupos.

Os dados encontrados nesses estudos foram corroborados por um ensaio clínico randomizado efetuado por Kruger et al. ${ }^{28}$, que teve como objetivo avaliar se a administração contínua de estatina, em pacientes que já faziam uso do medicamento, influenciaria a progressão ou a regressão da sepse quanto aos parâmetros de inflamação ou disfunção de órgãos em organismos em quadro séptico. Os indivíduos selecionados $(\mathrm{n}=150)$ foram divididos em dois grupos: um que continuaria fazendo uso de estatina na dosagem de $20 \mathrm{mg} / \mathrm{dia}$ e o grupo placebo, que teria a interrupção da medicação. $\mathrm{O}$ estudo teve duração de 28 dias e periodicamente os pacientes eram acompanhados por meio de exames clínicos e laboratoriais. A descoberta principal é que a continuação da terapia com estatina não foi associada à atenuação da resposta inflamatória ou à diminuição da falha de órgãos durante internação. Inversamente, a interrupção das estatinas não foi associada a uma recuperação inflamatória e piora na disfunção de órgãos. Também não houve diferenças significativas entre os 
dois grupos na taxa de mortalidade e no declínio da sepse. Os autores ainda ressaltam alguns fatores a serem considerados, como a possibilidade da dose de medicamento utilizada no estudo ter representado uma alteração das doses habituais de alguns pacientes; além de que, os efeitos pleiotrópicos possam ter persistido em alguns usuários prévios, mesmo após interrupção temporária do fármaco.

É importante ressaltar que nenhum estudo apresentado relatou efeitos colaterais ou intolerância às estatinas, no entanto não se pode desconsiderar esses efeitos quando esse grupo de fármacos é utilizado em pacientes graves com sepse $\mathrm{e}^{7,28,35}$. Apesar dos potenciais efeitos adversos mais importantes, tais como hepatotoxidade, neuropatias e rabdomiólise, serem considerados extremamente $\operatorname{raros}^{36,37}$, as concentrações plasmáticas elevadas atingidas durante a continuação do tratamento com esse medicamento demanda algum cuidado e atenção. Além disso, sua relação risco/benefício deve ser cuidadosamente avaliada $^{32,38}$, pois no paciente crítico são administrados simultaneamente vários medicamentos que podem interferir na biodisponibilidade das estatinas, aumentando os níveis séricos e reforçando sua toxicidade $^{38}$. Deve-se considerar também que organismos sépticos apresentam-se muitas vezes enfraquecidos, debilitados, com defesas imunológicas insuficientes ou com disfunção de órgãos, o que poderia facilitar a ocorrência de efeitos secundários indesejáveis?

\section{CONCLUSÃO}

Apesar de alguns estudos considerarem o uso de estatinas em pacientes sépticos uma teoria promissora, até o momento a maioria não demonstrou evidências sólidas e significativas quanto à redução de mortalidade e morbidade no quadro séptico. Muitos resultados não se sustentam quando são aplicados métodos estatísticos que levam em conta diferentes variáveis como idade, sexo, comorbidades e gravidade da doença. Esses fatores indicam, portanto, que seu efeito benéfico e protetor ainda não foi plenamente delimitado, permanecendo seu uso como arsenal terapêutico na sepse questionável, necessitando-se ainda de mais estudos clínicos para confirmar ou descartar as evidências até agora encontradas.

\section{REFERÊNCIAS}

1. Martin GS, Mannino DM, Eaton S, Moss M. The epidemiology of sepsis in the United States from 1979 through 2000. N Engl J Med. 2003;348(16): 1546-54.

http://dx.doi.org/10.1056/NEJMoa022139

2. Hospital Sírio Libanês [Internet]. Diagnóstico e tratamento precoces da sepse em adulto: protocolo institucional. Disponível em: http://www.hospitalsiriolibanes.org.br/sociedade-beneficentesenhoras/Documents/protocolos-institucionais/protocolo-sepse. pdf. Acesso em: 27 jun. 2013

3. Usinger J, Kazama H, McDonough JS, Griffith TS, Hotchkiss RS, Ferguson TA. Sepsis-induced apoptosis leads to active suppression of delayed-type hypersensitivity by $\mathrm{CD} 8^{+}$regulatory $\mathrm{T}$ cells trough a TRAIL-dependent mechanism. J Immunol. 2010;184(12):6766-72. http://dx.doi.org/10.4049/jimmunol.0904054

4. Remick DG. Pathophysiology of sepsis. Am J Pathol. 2007:170(5):1435-44 http://dx.doi.org/10.2353/ajpath.2007.060872

5. de Mendonça A, Vincent JI, Suter PM, Moreno R, Dearden NM, Antonelli $\mathrm{M}$, et al. Acute renal failure in the ICU: risk factors and outcome evaluated by the SOFA score. Intensive Care Med. 2000;26(7):915-21.

http://dx.doi.org/10.1007/s001340051281

6. Brivet FG, Kleinknecht DJ, Loirat P, Landais PJ. Acute renal failure in intensive care units: causes, outcome, and prognostic factors of hospital mortality; a prospective, multicenter study. French Group on Acute Renal Failure. Crit Care Med. 1996;24(2):192-8.

http://dx.doi.org/10.1097/00003246-199602000-00003

7. Ritt LEF, Guimarães HP, Avezum A, Piegas LS. Statins on sepsis: a new therapeutic gun in intensive care medicine? Rev Bras Ter Intensiva. 2006;18(4):402-6.

http://dx.doi.org/10.1590/S0103-507X2006000400014

8. Williams JM, Greenslade JH, Chu K, Brown AF, Paterson D, Lipman J. Prior statin use is not associated with improved outcome in emergency patients admitted with infection: a prospective observational study. Acad Emerg Med. 2011;18(2):127-34. http://dx.doi.org/10.1111/j.1553-2712.2010.00976.x
9. Raetz CR. Biochemistry of endotoxins. Annu Rev Biochem. 1990:59:129-70

http://dx.doi.org/10.1146/annurev.bi.59.070190.00102

10. Flo TH, Halaas O, Torp S, Ryan L, Lien E, Dybdahl B, et al Differential expression of Toll-like receptor 2 in human cells. J Leukoc Biol. 2001;69(3):474-81.

11. Dybdahl B, Wahba A, Lien E, Flo TH, Waage A, Qureshi N, et al. Inflammatory response after open heart surgery: release of heart-shock protein 70 and signaling through toll-like receptor-4. Circulation. 2002;105(6):685-90.

http://dx.doi.org/10.1161/hc0602.103617

12. Schor N. Acute renal failure and the sepsis syndrome. Kidney Int 2002;61(2):764-76 http://dx.doi.org/10.1046/j.1523-1755.2002.00178.x

13. Boechat AL, Boechat NO. Sepse: diagnóstico e tratamento. Rev Bras Clin Med São Paulo. 2010;8(5):420-7.

14. Bone RC, Grodzin CJ, Balk RA. Sepsis: a new hypothesis for pathogenesis of the disease process. Chest. 1997;112(1):235-43. http://dx.doi.org/10.1378/chest.112.1.235

15. Pober JS, Bevilacqua MP, Mendrick DL, Lapierre LA, Fiers W, Gimbrone MA Jr. Two distinct monokines, interleukin 1 and tumor necrosis factor, each independently induce biosynthesis and transient expression of the same antigen on the surface of cultured human vascular endothelial cells. J Immunol. 1986;136(5):1680-7.

16. Takemoto M, Liao JK. Pleiotropic effects of 3-hidroxy-3methylglutaryl coenzyme A reductase inhibitors. Arteriocler Thromb Vasc Biol. 2001;21(11):1712-9. http://dx.doi.org/10.1161/hq1101.098486

17. Rosenson RS, Tangney CC. Antiatherothrombotic properties of statins: implications for cardiovascular event reduction. JAMA. 1998;279(20):1643-50. http://dx.doi.org/10.1001/jama.279.20.1643

18. Schonbeck U, Libby P. Inflammation, immunity, and HMG-CoA reductase inhibitors: satins as antiinflamatory agents? Circulation. 2004;109(21 Suppl 1):II18-26. http://dx.doi.org/10.1161/01.CIR.0000129505.34151.23 
19. Issacsohn J, Setaro JF, Nicholas C, Davey JA, Diotalevi LJ, Christianson DS, et al. Effects of lovastatin therapy on plasminogen activator inhibitor-1 antigen levels. Am J Cardiol. 1994;74(7):735-7.

http://dx.doi.org/10.1016/0002-9149(94)90322-0

20. Sakamoto K, Sakamoto T, Ogawa H. The effect of 6 months of treatment with pravastatin on serum adiponection concentrations in Japanese patients with coronary artery disease and hypercholesterolemia: a pilot study. Clin Ther. 2006;28(7):1012-21. http://dx.doi.org/10.1016/j.clinthera.2006.07.001

21. Farmer JA. Pleiotropic effects of statins. Curr Atheroscler Rep. 2000;2(3):208-17. http://dx.doi.org/10.1007/s11883-000-0022-3

22. Ridker PM, Rifai N, Clearfield M, Downs JR, Weis SE, Miles JS, et al. Measurement of $\mathrm{C}$-reactive protein for the targeting of statins therapy in the primary prevention of acute coronary events. N Engl J Med. 2001;344(26):1959-65.

http://dx.doi.org/10.1056/NEJM200106283442601

23. Dimmeler S, Aicher A, Vasa M, Mildner-Rihm C, Adler K, Tiemann $M$, et al. HMG-CoA reductase inhibitors (statins) increase endothelial progenitor cells via the PI 3-kinase/Akt pathway. J Clin Invest. 2001;108(3):391-7.

http://dx.doi.org/10.1172/JCl200113152

24. Terada $Y$, Inoshita S, Nakashima O, Yamada T, Kuwahara M, Sasaki S, et al. Lovastatin inhibits mesangial cell proliferation via p27Kip1. J Am Soc Nephrol. 1998;9(12):2235-43.

25. Sparrow CP, Burton CA, Hernandez M, Mundt S, Hassing H, Patel S, et al. Simvastatin has anti-inflamatory and antiatherosclerotic activities independent of plasma cholesterol lowering. Arterioscler Thromb Vasc Biol. 2001;21(1):115-21. http://dx.doi.org/10.1161/01.ATV.21.1.115

26. Zoja C, Corna D, Rottoli D, Cattaneo D, Zanchi C, Tomasoni S, et al. Effect of combining ACE inhibitor and statin in severe experimental nephropathy. Kidney Int. 2002;61(5):1635-45. http://dx.doi.org/10.1046/j.1523-1755.2002.00332.x

27. Novak V, Eisinger M, Frenkel A, Terblanche M, Adhikari NK, Douvdevani $A$, et al. The effects of statin therapy on inflammatory cytokines in patients with bacterial infections: a randomized double-blind placebo controlled clinical Trial. Intensive Care Med. 2009;35(7):1255-60.

http://dx.doi.org/10.1007/s00134-009-1429-0

28. Kruger PS, Harward ML, Jones MA, Joyce CJ, Kostner KM, Roberts MS, et al. Continuation of statin therapy in patients with presumed infection a randomized controlled trial. Am J Respir Crit Care Med. 2011;183(6):774-81.

http://dx.doi.org/10.1164/rccm.201006-09550C

29. Nseir W, Mograbi J, Abu-Elheja O, Bishara J, Assy N. The impact of prior long-term versus short-term statin use on the mortality of bacteraemic patients. Infection. 2012;40(1):41-8. http://dx.doi.org/10.1007/s15010-011-0190-9

30. Patel JM, Snaith C, Thickett DR, Linhartova L, Melody T, Hawkey P, et al. Randomized double-blind placebo-controlled trial of 40 $\mathrm{mg} /$ day of atorvastatin in reducing the severity of sepsis in ward patients (ASEPSIS Trial). Crit Care. 2012;16(6):R231. http://dx.doi.org/10.1186/cc11895

31. Donnino MW, Cocchi MN, Howell M, Clardy P, Talmor D, Cataldo L, et al. Statin therapy is associated with decreased mortality in patients with infection. Acad Emerg Med. 2009;16(3):230-4. http://dx.doi.org/10.1111/j.1553-2712.2009.00350.x

32. Mekontso Dessap A, Ouanes I, Rana N, Borghi B, Bazin C, Katsahian $\mathrm{S}$, et al. Effects of discontinuing or continuing ongoing statin therapy in severe sepsis and septic shock: a retrospective cohort study. Crit Care. 2011;15(4):R171. http://dx.doi.org/10.1186/cc10317

33. Williams JM, Greenslade JH, Chu K, Brown AF, Paterson D, Lipman J. Prior statin use is not associated with improved outcome in emergency patients admitted with infection: a prospective observational study. Acad Emerg Med. 2011;18(2):127-34 http://dx.doi.org/10.1111/j.1553-2712.2010.00976.x

34. Leung S, Pokharel R, Gong MN. Statins and outcomes in patients with bloodstream infection: a propensity-matched analysis. Crit Care Med. 2012;40(4):1064-71. http://dx.doi.org/10.1097/CCM.0b013e31823bc9e7

35. Knopp RH. Drug treatment of lipid disorders. N Engl J Med. 1999;341(7):498-511.

36. Baigent C, Keech A, Kearney PM, Blackwell L, Buck G, Pollicino C, et al. Efficacy and safety of cholesterol-lowering treatment: prospective meta-analysis of data from 90,056 participants in 14 randomised trials of statins. Lancet. 2005;366:(9493):1267-78. http://dx.doi.org/10.1016/S0140-6736(05)67394-1

37. Tolman KG. The liver and lovastatin. Am J Cardiol. 2002; 89(12):1374-80. http://dx.doi.org/10.1016/S0002-9149(02)02355-X

38. Carrillo-Esper R, Rivera-Buendía S, Carrillo-Córdova JR, CarrilloCórdova LD. Estatinas, ¿alternativa terapéutica en sepsis? Gac Méd Méx. 2007;143(6):499-3. 